\title{
On the influence of inhibitory STDP on balanced state random networks
}

\author{
Felix Effenberger ${ }^{1 *}$, Anna Levina ${ }^{1,2}$, Jürgen Jost ${ }^{1}$ \\ From Twenty Second Annual Computational Neuroscience Meeting: CNS*2013 \\ Paris, France. 13-18 July 2013
}

The distribution of synaptic efficacies in neural networks takes fundamental influence on their dynamics and the modification of synaptic strengths forms the foundation of learning and memory. A prominent plasticity rule that has been observed in vitro is spike-timing-dependent plasticity (STDP). While first studied in glutamatergic synapses, recently also STDP of GABAergic synapses came into the focus of experimental and theoretical research [1].

We study random balanced state networks of leaky integrate-and-fire neurons in the asynchronous irregular (AI) regime [2] that is believed to be a good theoretical fit to the activity of cortical networks in vivo. We consider driven networks that receive Poisson input as well as networks in a self-sustained state of activity. In order to assess the influence of excitatory and inhibitory
STDP on the network dynamics, we introduce these two plasticity rules independently, observing network dynamics and weight distributions after a transient phase. Note that both additive and multiplicative STDP rules yield the same network dynamics as described below.

When introducing excitatory STDP alone, parameters involving the maximal weight have to be fine-tuned in order to keep the network activity stably in the AI regime [3]. For almost all parameter values the network activity becomes unstable, leaving the AI regime and settling in a pathological, highly synchronized state with saturated firing rates of most cells, see Figure 1A. We also observed that even without STDP, few strong excitatory connections can substantially destabilize network dynamics yielding pathological states. Interestingly, this
A

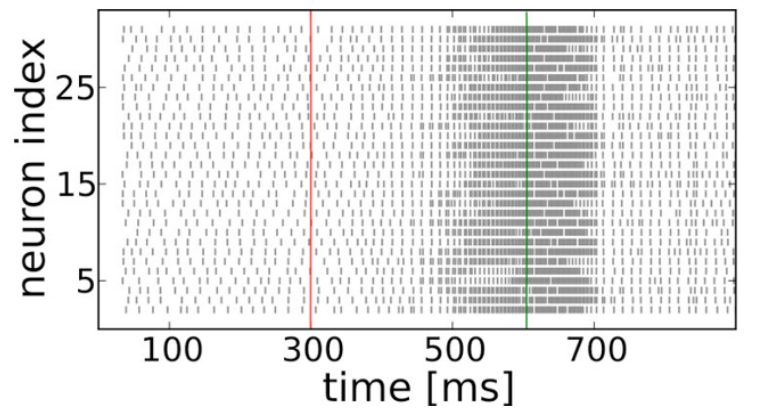

B

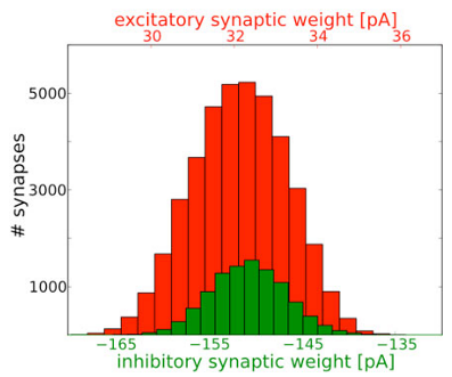

Figure 1 A. Raster plot of 30 randomly sampled cells showing network activity. Red line: activation of excitatory STDP, green line: activation of inhibitory STDP. B. Weight distributions of plastic synapses converging onto 100 randomly sampled excitatory neurons. Red: excitatory synapses, green inhibitory synapses.

\footnotetext{
* Correspondence: Felix.Effenberger@mis.mpg.de

${ }^{1}$ Max-Planck-Institute for Mathematics in the Sciences, Leipzig, 04103,

Germany

Full list of author information is available at the end of the article
} 
destabilization does not happen when in addition to excitatory STDP we also introduce STDP for inhibitory synapses projecting onto excitatory cells. The latter setup results in a network that stably rests in the AI regime, see Figure 1A. Both STDP rules yield nearGaussian distributions of synaptic weights, see Figure 1B. Inhibitory STDP even manages to stabilize a network that was brought to a pathological state by excitatory STDP, see Figure 1A. This clearly shows that inhibitory STDP has a stabilizing effect on network dynamics and we expect that especially in combination with synaptic scaling and in the context of clustered networks [4] other non-trivial dynamical effects will become visible.

\section{Acknowledgements}

The second author acknowledges support by the Federal Ministry of Education and Research (BMBF) Germany under grant number 01GQ1005B.

\section{Author details}

${ }^{1}$ Max-Planck-Institute for Mathematics in the Sciences, Leipzig, 04103, Germany. ${ }^{2}$ Bernstein Center for Computational Neuroscience Göttingen, 37077, Germany.

Published: 8 July 2013

\section{References}

1. Vogels TP, Sprekeler H, Zenke F, Clopath C, Gerstner W: Inhibitory plasticity balances excitation and inhibition in sensory pathways and memory networks. Science 2011, 334:1569-73, (New York, NY).

2. Brunel N: Dynamics of sparsely connected networks of excitatory and inhibitory spiking neurons. Journal of computational neuroscience 2000, 8:183-208.

3. Morrison A, Aertsen A, Diesmann M: Spike-timing-dependent plasticity in balanced random networks. Neural computation 2007, 19:1437-67.

4. Litwin-Kumar A, Doiron B: Slow dynamics and high variability in balanced cortical networks with clustered connections. Nature Neuroscience 2012, 15:1498-1505.

doi:10.1186/1471-2202-14-S1-P200

Cite this article as: Effenberger et al:: On the influence of inhibitory STDP on balanced state random networks. BMC Neuroscience 201314 (Suppl 1):P200.

\section{Submit your next manuscript to BioMed Central} and take full advantage of:

- Convenient online submission

- Thorough peer review

- No space constraints or color figure charges

- Immediate publication on acceptance

- Inclusion in PubMed, CAS, Scopus and Google Scholar

- Research which is freely available for redistribution

Submit your manuscript at www.biomedcentral.com/submit 